\title{
Global Health and Tropical Medicine in the Twenty-First Century: A Renewed Interest in the Understanding and the Control of Helminth Infections
}

\author{
Rojelio Mejia $^{1}$ - Fabrizio Bruschi ${ }^{3,4}$ - Maria Elena Bottazzi ${ }^{1,2}$
}

Published online: 30 September 2015

(C) Springer International Publishing AG 2015

\begin{abstract}
The twenty-first century has shown an expansion and renewed excitement in global health, and tropical medicine is one of the critical drivers leading this global health phenomenon. Among the tropical diseases, helminth infections rank first in the list of diseases causing the highest disability adjusted life years. Because of the importance of helminth infections and their impact on global health, we highlight in this commentary an elegant compilation of reviews recently published by Springer, which provide a detailed overview of the biology, pathology, diagnosis, treatment, and prevention strategies for the helminths that can cause human disease and morbidity.
\end{abstract}

Keywords Filariasis $\cdot$ Soil-transmitted helminths ·

Strongyloides $\cdot$ Cysticercosis $\cdot$ Toxocariasis

This article is part of the Topical Collection on Invited Commentary on Global Health and Tropical Medicine

Rojelio Mejia

rmejia@bcm.edu

Fabrizio Bruschi

fabrizio.bruschi@med.unipi.it

Maria Elena Bottazzi

Bottazzi@bcm.edu

1 Sabin Vaccine Institute and Texas Children's Hospital Center for Vaccine Development, National School of Tropical Medicine, Baylor College of Medicine, Houston, TX, USA

2 Department of Biology, Baylor University, Waco, TX, USA

3 Dipartimento di Ricerca Traslazionale, NTMC Università di Pisa, Pisa, Italy

4 Collaborative Member, National School of Tropical Medicine, Baylor College of Medicine, Houston, TX, USA

\section{Main Text}

The twenty-first century has shown an expansion and renewed excitement in global health. This has been especially driven by health professionals and students who are calling for training programs that can prepare them to work in this global environment. In response, many academic institutions since the early 2000 s have attempted to define and understand the term of global health and how it is differentiated from other long-lasting educational programs, for instance in the field of public health. This has led to the implementation of a variety of new global health programs worldwide with the ultimate goal to train, educate a new generation of health professional, and advance new scientific knowledge and novel research discoveries such that it can significantly lead towards the improvement of the health of the "bottom billion" or the poorest populations in the world. Macfarlane et al., in 2008, wrote an elegant review of the trends and efforts by academic institutions towards global health [1].

Tropical medicine is one of the critical drivers leading this global health phenomenon. It builds on the history established during the turn of the twentieth century by Western European institutions such as early discoveries of parasite life cycles where many of the early drugs and diagnostics developments still in use today were spearheaded $[2,3]$. This century has now seen an expansion of centers of excellence within the Americas including the creation in 2011 of the National School of Tropical Medicine at Baylor College of Medicine in Houston Texas $[2,3]$. These academic and research institutions continue to lead enormous efforts into the understanding of tropical diseases, their burden of disease, their distribution, and their interplay with global health. These efforts have been highlighted and published in the Global Burden of Disease Study 2010, where helminth infections rank first in the list contributing to the highest disability adjusted life year estimations [4]. In response 
to these very important findings, a group of renowned scientists prepared a compilation of reviews that explore the latest advances in the wide vastness in the world of helminths. This compilation, published by Springer in 2014 and entitled "Helminth Infections and their impact on Global Public Health," clearly highlights how our world is still a "Wormy World" (as quoted in 1947 by Norman Stoll) [5].

This first edition covers in-depth information of the systematics and biology of helminths, the paleoparasitology of helminth infections, and for the most common helminth infections a description of the etiological agent, the epidemiology, pathogenesis, immunology, and most importantly the advances in the current tools for diagnosis, prevention, and treatment.

To highlight a few novel aspects of this compilation, we will briefly recap selected chapters that we found of particular importance to global health and the tropical medicine field:

The chapter on "lymphatic and tissue filariasis" by Mark P. Hubner, Laura E. Layland, and Achim Hoerauf (a very wellknown group in the field) represents an exhaustive overview about filarial agents which are still a serious public health concern not only in Africa. Just as an example, India has 553 million people at risk for lymphatic filariasis. After discussing both the similarities and differences between these two types of filariasis, as regards over all host immune reactions which have relevant implications in the pathogenesis of the disease, the authors have added an interesting paragraph on alterations in clinical manifestations of immunocompromised individuals infected with filariae. In the treatment section, they have given particular emphasis on the new approach of treatment of some of these diseases, targeting the endosymbiotic Wolbachia bacterium with antibiotics, which has a dramatic microfilaricidal effect. The chapter ends with an updated paragraph on prevention and control measures.

A key message given by the authors is represented by the passage "...... society should not stop trying to develop vaccines since the overall effectiveness of mass drug administration in several regions is worrying due to noncompliance or potential drug resistance."

The chapter on "soil-transmitted helminthiasis" by Albis F. Gabrielli, Antonio Montresor, and Lorenzo Savioli (leading experts from the World Health Organization) provides an indepth overview of the morbidity caused by Ascaris lumbricoides, Trichuris trichiura, Necator americanus, and Ancylostoma duodenale. The authors provide an in-depth assessment of the disease burden and the populations at risk or currently infected populations (which amount to 300 million to 1 billion worldwide). The authors also provide an overview of the epidemiology, risk factors, immunology, and clinical manifestations. Interestingly, the authors note that diagnosis still relies primarily on microscopic techniques based on identification of eggs or larvae in stool, and use of semiquantitative techniques, which are used to establish the intensity of worm burden infections. For treatment, the authors describe the World Health Organization recommendations for clinical management but also describe a control strategy called "preventive chemotherapy" that has as an ultimate goal to reduce morbidity within pre- and school-age children with very detailed global goals and targets. The chapter finalizes with a key message that control for soil-transmitted helminthes will only be achieved by applying an integrated approach, where the authors provide practical case studies and examples as well as the development of complementary approaches such as health education, improved sanitation, and the development of other control tools such as preventive vaccines.

The chapter on "Strongyloides stercoralis and Strongyloidosis" by Masataka Korenaga and Fabrizio Bruschi reviews the life-cycle, larval mechanism of development, diagnosis, and current first-line or alternative therapies for the more prevalent $S$. stercoralis and less common Strongyloides fuelleborni. Infections with Strongyloides spp. have been grossly underestimated with current reports of 100 million people infected, making this one of the most neglected of the neglected tropical diseases.

The Strongyloides spp. life-cycle differs than most nematodes, in that the second stage larvae can convert to L3i (the infectious filariform stage) and can penetrate the intestinal mucosa, restarting the life-cycle without an environmental stage. It is this unique mechanism that increases the burden of disease for strongyloidiosis with chronic infections lasting decades in affected individuals.

A vital review of this chapter is the clinical implications for immunocompromised patients. While strongyloidiosis is auto-infectious, it generally is clinically quiescent with symptoms ranging from asymptomatic to abdominal pain, diarrhea, and urticarial or larva currens. What makes strongyloidiosis such a malignant disease is when the host has an acquired immunocompromised challenge either iatrogenic via corticosteroids (or other immunosuppressives) or infected individuals with human T-lymphotropic virus-1 (HTLV-1). This leads to a depression in cell-mediated immunity and increases the risk of hyperinfection and dissemination of larvae that can migrate to organs beyond the typical pulmonary/ gastrointestinal tract. Symptoms are severe and often complicated by sepsis due to gram-negative bacteria that accompany the larvae as they migrate out of the intestines.

The chapter is complete with an informative review of sensitivity/specificity of diagnostic assays, including enzyme-linked immunosorbent assay (ELISA) which uses the recombinant protein NIE, as antigen, luciferase immunoprecipitation systems (LIPS), real-time polymerase chain reaction (PCR), and nested PCR. Treatment is divided into firstline therapy, alternative, and prevention.

The chapter on "taeniosis and cysticercosis" by Elizabeth Ferrer and Teresa Gárate is appropriately divided in the two different clinical presentations of Taenia saginata and Taenia solium (Taenia asiatica is briefly described). It begins by 
describing the differences of taeniasis versus cysticercosis and its clinical presentations. These tapeworms continue to cause significant human morbidity and livestock industry loses.

The host immune response is divided into innate and adaptive responses with more data on the cellular response due to neurocysticercosis. Interestingly, the parasite surface can attach host antibodies and complement, allowing the parasite to propagate and avoid an inflammatory response. As mentioned in this chapter, cysticerci generally lead to an asymptomatic infection for many years until the dying parasite loses its immunomodulating properties. In neurocysticercosis, this leads to seizures or the need for emergent neurosurgery, depending on anatomical presence of the cysticerci.

Diagnosis is covered by describing biopsy for cysticerci or by stool microscopy for taeniosis. Immunodiagnosis is done using an ELISA using Taenia crude extract with a secondary antibody to anti-excretory/secretory antigen. Diagnosis is complete by emphasizing the critical use of computed axial tomography (CT) and magnetic resonance imaging (MRI). The key parts of this chapter are the review on treatments for taeniosis, for which a single dose of praziquantel is needed. More importantly is the treatment and prognosis of neurocysticercosis and how management should be individualized. Therapy depends on the size, number, and location of cysts in the central nervous system.

The chapter on "toxocariasis" by Clare M. Hamilton, Ayako Yoshida, Elena Pinelli, and Celia V. Holland is focused on infections caused by two parasites of dogs (Toxocara canis) and cats (Toxocara cati), which have an ubiquitous distribution and incidentally infect humans who present with a number of clinical syndromes including visceral larva migrans, ocular larva migrans, and neurotoxocarosis.

Of particular interest in the chapter is the review of seroprevalence studies, which indicate at global level a high human exposure. However, the general public is not aware of the risks of infection with such parasites and toxocarosis may be considered a classic neglected disease.

A stimulating section of the chapter is devoted to the evaluation of commercially available serodiagnostic kits, which provides to the professionals a useful tool to make a good choice.

In conclusion, we invite the reader to review this useful resource and learn about the advances in research and development of helminth infections and how these pivotal studies and underpinning provide an impact on global public health.

\section{References}

1. Macfarlane SB, Jacobs M, Kaaya EE. In the name of global health: trends in academic institutions. J Public Health Policy. 2008;29:383401.

2. Hotez P. A national school of tropical medicine and neglected infections of poverty in North America. Public Libr Sci Negl Trop Dis. 2010;4(6):E735.

3. Hotez P. Tropical medicine in the horse latitudes. Curr Trop Med Rep. 2014;1(1):3-5.

4. Hotez PJ, Alvarado M, Basanez, MG, et al. The Global Burden of Disease Study 2010: interpretation and implications for the neglected tropical diseases

5. Bruschi $\mathrm{F}$ (Editor). Helminth infections and their impact on global public health. Springer-Verlag Wien. 2014 First Edition 